Wissenschaftler bleiben in ihren Aussagen zu spezifischen Aspekten depressiver Erkrankungen ebenfalls vage.

Dagegen werden eindrückliche Einblicke in Grenzbereiche des modernen Wissenschaftsbetriebs und in die Denkwelten der an diesen Fronten tätigen Wissenschaftler vermittelt. "Wir sind gerade dabei, unser Menschsein zu verändern", sagt seelenruhig der Molekulargenetiker Georg Church, der fast so aussieht, wie man sich als Kleinkind den lieben Gott vorstellt. «Aber wir sind keine Übermenschen. Wir sind nur Ingenieure, die ihren Job machen, wie Ingenieure dies immer getan haben.» Der Supercomputer "MareNostrum» des Nationalen Genom-Analyse-Zentrums in Barcelona, das vom Schweizer Ivo Gut geleitet wird, steht bezeichnenderweise in einer ehemaligen Kathedrale.

Auch wenn man sich mitunter fragt, ob der Protagonist die Antworten auf seine Fragen zur Depression am richtigen Ort sucht, ist seine filmische Reise in vielerlei
Hinsicht bereichernd. Zu dieser Gesamtbilanz tragen auch die Bilder bei, die die Filmemacher finden, um die komplexen biochemischen und molekularbiologischen Vorgänge im Gehirn zu visualisieren, und die eine eigenartige, mitunter fast mystische Stimmung erzeugen. Dass sie die Pfade des Wissenschaftsbetriebs verschiedentlich verlassen und Künstler aufspüren, die Erkenntnisse aus der Molekularbiologie und Genetik in Musik oder Plastiken transformieren, erweitert den Film um eine wesentliche Dimension.

Die intensivsten Momente entstehen aber interessanterweise, wenn Frank Schauder ganz konventionell vor der Kamera davon erzählt, wie er seine Depression erlebt hat. In diesen Einstellungen sind sein Schmerz, seine Verzweiflung, seine Verlassenheit unmittelbar spürbar und man vermeint als Zuschauer, eine Ahnung davon zu erhalten, was es bedeutet, in einer Depression gefangen zu sein.

Das dunkle Gen läuft ab 28. Mai in den Schweizer Kinos.

\title{
Wie Transgender-Patienten betreuen?
}

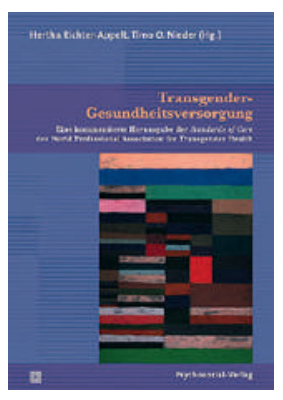

Hertha Richter-Appelt, Timo O. Nieder (Hg.)

Transgender-Gesundheitsversorgung

Eine kommentierte Herausgabe der Standards of Care der World Professional Association for Transgender Health

Giessen: Psychosozial-Verlag; 2014.

203 Seiten. 24.90 EUR

ISBN 978-3-8379-2424-4

Seit 1979 gibt die interdisziplinäre World Professional Association for Transgender Health (WPATH) Empfehlungen zur Betreuung von Menschen heraus, die an einer fehlenden Übereinstimmung ihrer subjektiven Geschlechtszugehörigkeit mit ihren geschlechtsspezifischen körperlichen Merkmalen leiden. Hertha Richter-Appelt und Timo O. Niederer, zwei anerkannte Spezialisten auf diesem Gebiet, haben die 7. Version der Vorsorgeempfehlungen aus dem Jahr 2011 aus dem Englischen übersetzt und kommentiert.

Erklärtes Ziel ist, den Patienten zu einer Linderung ihres Leidensdrucks, zu Zufriedenheit mit ihrer Identität und zu optimaler Selbstverwirklichung zu verhelfen. In diesem Zusammenhang wird gefordert, allen Patienten den Zugang zu entsprechenden psychischen, hormonellen und operativen Behandlungsmassnahmen zu ermöglichen. Gleichzeitig soll für keine der genannten Therapien ein Zwang bestehen; vielmehr soll ein individuell den Bedürfnissen des Patienten angepasster Behandlungsplan erarbeitet werden. Die einzelnen Behandlungsmethoden mit Zulassungskriterien und Kontraindikationen werden übersichtlich diskutiert.

Die Ausführungen beziehen sich auf den westlichen Kulturkreis. Allerdings stösst die Übertragbarkeit auf schweizerische Verhältnisse an Grenzen, insbesondere was den Vorschlag des Einfrierens von Eizellen betrifft, um nach einer Frau-zu-Mann-Operation nachträglich Nachkommen mittels Leihmutterschaft zu ermöglichen. Und bezüglich der Bedürfnisse von intersexuellen Menschen, deren Genitale bei Geburt uneindeutig ist oder deren körperliche sexuelle Entwicklung atypisch verläuft, wird auf separate Guidelines verwiesen. Für diejenigen Ärzte aber, die Patienten mit Transsexualität und Genderdysphorie betreuen, handelt es sich um ein gut strukturiertes, einfach lesbares und mit 203 Seiten überschaubares Werk.

Erika Nussberger, Vevey 